\title{
PTPRO Promotes Oxidized Low-Density Lipoprotein Induced Oxidative Stress and Cell Apoptosis through Toll-Like Receptor 4/Nuclear Factor кB Pathway
}

\author{
Caihong Liang ${ }^{a, b}$ Xiaochen Wang ${ }^{c}$ Jianping $\mathrm{Hu}^{\mathrm{d}}$ Xiaoging Lian ${ }^{\mathrm{b}}$ Tiantian Zhu ${ }^{\mathrm{b}}$
} Hui Zhang ${ }^{d}$ Ning Gu ${ }^{a, e}$ Jun Li ${ }^{d}$

\begin{abstract}
aNanjing University of Chinese Medicine, Nanjing, bepartment of cardiovascular, The Affiliated Jiangning Hospital of Nanjing Medical University, Nanjing, 'Key Laboratory on Living Donor Liver Transplantation, Ministry of Health, Department of Liver surgery, Collaborative Innovation Center for Cancer Personalized Medicine, First Affiliated Hospital of Nanjing Medical University, Nanjing, dDepartment of General Surgery, The Affiliated Jiangning Hospital of Nanjing Medical University, Nanjing, ${ }^{e}$ Department of cardiovascular, The Third Affiliated Hospital of Nanjing University of Chinese Medicine, Nanjing, P.R. China
\end{abstract}

\section{Key Words}

Atherosclerosis $\cdot \mathrm{Ox}-\mathrm{LDL} \cdot$ Macrophage Protein tyrosine phosphatase receptor type O

\begin{abstract}
Background/Aims: Critical roles of phosphatase receptor type O (PTPRO) and toll-like receptor 4 (TLR4) have been implicated in inflammation. However, little is known about their functional effects on atherosclerosis (AS). We aim to study their potential function in AS. Methods: An oxidized low-density lipoprotein (ox-LDL) induced AS model constructed with PTPRO overexpressing RAW264.7 cells and PTPRO knockout macrophages. Cell apoptosis was assayed by flow cytometry and fatty accumulation was evaluated by oil red staining. The production of ROS (reactive oxygen species), SOD (superoxide dismutase), MDA (malondialdehyde), TC (Triglyceride), and TG (total cholesterol) was evaluated. Western blot was performed to detect the expression of CD36, TLR4 and nuclear factor kB (NF-kB). Results: PTPRO expression was promoted in a dose-dependent and time-dependent manner following ox-LDL challenging. In PTPRO-over-expressing cells, CD36 expression and the level of oil-red staining, TC and TG were increased; ROS production, MDA and level of cell apoptosis were improved, but SOD was reduced. However, in PTPRO knockout cells opposite results were found. TLR4 and NF-KB/p65 phosphorylation was significantly enhanced in PTPRO over-expressing cells, while significantly down-regulated in PTPRO knockout cells. Conclusion: PTPRO plays ital roles in AS via promoting ox-LDL induced oxidative stress and cell apoptosis through TLR4/NF-KB pathway.

C. Liang and X. Wang contribute equally to this study.




\section{Introduction}

Atherosclerosis (AS) is the leading cause of diverse infaust vascular events, including coronary artery disease, peripheral artery disease and stroke. With the increasing worldwide prevalence, it accounts for the most cardiovascular morbidity and mortality [1, 2]. It has been generally established that AS is an inflammatory disease involving a chronic persistent state. AS commonly initiates within the arterial wall primarily as a result of the destruction occurring in endogenously structures, which can be attributed to several matters, such as hypertension, smoking, oxidized low-density lipoprotein (ox-LDL) and so forth [3-5]. OxLDL, as the most important factor contributing to the development of AS, can activate both the innate and adaptive immune responses with the differentiation from monocytes to macrophages and the unregulated phagocytic activity of macrophages [6-8], subsequently induce the accumulation of lipid and the formation of foam cells, which is the hallmark event of AS $[9,10]$.

Previous studies have provided abundant evidence for the pivotal roles of ox-LDL and macrophages in the genesis of AS [11-13]. Generally, overloaded ox-LDL in plasma would be scavenged by macrophages via scavenger receptors, notably the type A scavenger receptor (SRA) and a type B family member, CD36 [14], which results in the activation of macrophages and leading to the production of reactive oxygen species (ROS). Excessive ROS can cause the cellular damage through the detrimental modification of many vital macromolecules, including DNA, RNA, protein and lipids, which may ultimately result in cell metabolic disorder and macrophage apoptosis. Therefore, treatments focusing on the inhibition of the production of ROS induced by ox-LDL may be beneficial for the protection of macrophage activity and thus prevent and suppress the development of AS.

Phosphatase receptor type 0 (PTPRO) is one of the receptor types of phosphor tyrosine phosphatases (PTPs) [15]. As an integral membrane protein with its full isoform (PTPRO) expressed in many parenchymal cells (including lung, liver, and breast) and truncated isoform identified in inflammatory cells, such as macrophages and lymphocytes, PTPRO has been verified as a functional participator involved in the development of several tumors and inflammatory diseases [16-19]. Former studies suggested that PTPRO played critical roles in acute inflammation mediated by T lymphocytes [20] and macrophages [17] via activation of NF-kB signaling pathway. AS is a complicated inflammation associated disease, and in the occurrence and progression of AS, many vital molecules and inflammatory signaling pathways are activated, among which NF-kB pathway is of great significance.

Toll-like receptor 4 (TLR4) was formerly demonstrated to interact with PTPRO in diverse diseases $[17,18]$. TLR4 is a most intensively studied member of TLRs family, which is extensively expressed on macrophages. Previous studies have demonstrated that TLR4 was involved in the regulation of macrophage lipid accumulation [21-25], and it is essential for ox-LDL-induced lipid uptake in macrophages $[2,26]$.

Up to date, the role of PTPRO in AS has not been elucidated. Considering the significant role of TLR4 in AS, in combination with the interaction of TLR4 and PTPRO, we hypothesizing that PTPRO, as an important regulator of macrophage activity, may act as a fine turner in macrophage mediated ox-LDL induced AS.

\section{Materials and Methods}

Animals and cell lines

Male wild-type (WT) C57BL/6 mice were purchased from the Laboratory Animal Center of Nanjing Medical University. The PTPRO-knockout (KO) C57BL/6 mice were gifted by Dr. John L. Bixby (University of Miami, Miami, FL). All mice were maintained and bred under specific pathogen-free facilities in the Laboratory Animal Center of Nanjing Medical University. All experiments were carried out according to the Guide for the Care and Use of Laboratory Animals. RAW264.7 cell line (derived from murine macrophages) was purchased from the American Type Culture Collection (ATCC, Manassas, VA, USA)

\section{KARGER}




\section{Cellular Physiology Cell Physiol Biochem 2017;42:495-505 \\ \begin{tabular}{l|l|} 
and Biochemistry Published 10.1159/000477596 & $\begin{array}{l}\text { C } 2017 \text { The Author(s). Published by S. Karger AG, Basel } \\
\text { www.karger.com/cpb }\end{array}$
\end{tabular}}

Liang et al.: PTPRO and Atherosclerosis

In order to obtain primary macrophages, eight- to ten-week-old mice were intraperitoneally injected with mercaptoethanol (Gibco, Carlsbad, CA, USA) $(25 \mathrm{mg} / \mathrm{kg}$ ) for $24 \mathrm{~h}$. Then mice were sacrificed and ascites was collected and added to 6-well plates. For cell cultivation, Dulbecco's modified Eagle's medium (DMEM) medium (Invitrogen Corp., Grand Island, NY, USA) added with 10\% heat-inactivated fetal bovine serum (FBS) (Gibco, Carlsbad, CA, USA), $2 \mathrm{mM}$ L-glutamine, $100 \mathrm{U} / \mathrm{ml}$ penicillin, and $100 \mathrm{mg} / \mathrm{ml}$ streptomycin was used. Cells were maintained in $5 \% \mathrm{CO}_{2}$ at $37^{\circ} \mathrm{C}$.

Cell transfection

RAW264.7 cells $\left(0.5-1.0 \times 10^{6}\right)$ were transfected with PLV-PTPRO-GFP plasmid accompanied with polybrene reagent as previously described [13]. Clone ExpressTM II One Step Cloning Kit (Vazyme biotech co.,Itd.) was used to construct PLV-PTPRO-GFP plasmids according to the manufacturer's instruction. 293 T cells were used to packaging the PLV-PTPRO-GFP plasmids which were the source of the pressed protein. After $72 \mathrm{~h}, 293 \mathrm{~T}$ cell supernatant was collected and applied as the source of the pressed protein (PLVPTPRO-GFP).

\section{Cell co-culture and cell proliferation assay}

RAW264.7 cells were co-cultured with smooth muscle cell line, MOVAS, in an indirect co-culture system as described [27, 28]. Totally $2 \times 10^{5}$ RAW264.7 cells after $50 \mathrm{mg} / \mathrm{L} \mathrm{ox}$-LDL challenging for 24 hours were seeded in the up layer in the Transwell units, and $2 \times 10^{5}$ MOVAS cells were seeded in the down layer in the well of six-well plates (In Vitro Scientific, CA, USA). After 24 hours cultivation in DMEM medium added with 10\% FBS, MOVAS cells were collected and were treated with Cell-Light EdU Apollo567 In Vitro Kit (RiboBio, Guangzhou, China) according to the given instruction. Cell samples were detected with a laser confocal scanning microscopy (Carl Zeiss Jena, Germany).

\section{Real-time polymerase chain reaction (PCR)}

Total RNAs were extracted from cells with TRIzol reagent (Invitrogen) and then quantized. The integrity of the isolated RNA was checked using NanoDrop 2000/2000c (Thermo Scientific, MA, USA). In order to get cDNA, reverse transcriptase (TaKaRa, Tokyo, Japan) was used to reverse transcribe total RNAs (500 ng). Real-time PCR was performed using Step one plus machine (Applied Biosystems, Darmstadt, Germany) with SYBR Green Mastermix kit (Takara, Tokyo, Japan) and results were analyzed in triplicate assays. Primers used were listed in Table 1.

\section{Flow cytometry analysis}

Cells were incubated with or without $50 \mathrm{mg} / \mathrm{L}$ ox-LDL for $24 \mathrm{~h}$ after transfection. Then, Annexin V-FITC/ PI Apoptosis Detection Kit (Vazyme Biotech, Nanjing, China) were used to detect cell apoptosis in cells according to the instructions. Briefly, the cells were treated with tyrisin and collected, then washed twice with PBS. Cell pellet was suspended in 1x binding buffer $(100 \mu \mathrm{L})$, after which annexin APC $(5 \mu \mathrm{L})$ and PE $(5 \mu \mathrm{L})$ was added, then cells were gently vortexed and subsequently incubated for 10 min at room temperature in the dark. Flow cytometry analysis was conducted using a FACS Calibur flow cytometer equipped with CellQuest software (BD Biosciences, New York, NY, USA).

\section{Oil red $O$ staining}

Cells incubated with or without $50 \mathrm{mg} / \mathrm{L}$ ox-LDL (Unionbiol, Beijing, China) for $24 \mathrm{~h}$ after transfection were fixed with $4 \%$ paraformaldehyde for $10 \mathrm{~min}$. Samples were washed with PBS for twice, then stained with filtered Oil Red 0 solution (60\% Oil Red 0 dye and $40 \%$ water; Sigma, St. Louis, MO, USA) at room temperature for $15 \mathrm{~min}$. Samples were then washed for twice with PBS. Oil red staining was observed under a microscope (Olympus, Tokyo, Japan) and the intensity was measured by Image-Pro Plus 6.0.

Table 1. Primers used for real-time PCR

\begin{tabular}{ccc}
\hline Genes & Forward $\left(5^{\prime} \sim 3^{\prime}\right)$ & Reverse $\left(5^{\prime} \sim 3^{\prime}\right)$ \\
\hline PTPRO & GAGGTGAACCCTAACGTGGTG & TCATGGAGACTAGCCTCACCC \\
GAPDH & GCCTCGTCCCGTAGACAAAA & GATGGGCTTCCCGTTGATGA \\
\hline
\end{tabular}




\section{Cellular Physiology Cell Physiol Biochem 2017;42:495-505 \\ \begin{tabular}{l|l} 
and Biochemistry Published online: June 02, 2016 & $\begin{array}{l}\text { (c) } 2017 \text { The Author(s). Published by S. Karger AG, Basel } \\
\text { www.karger.com/cpb }\end{array}$
\end{tabular}}

Liang et al.: PTPRO and Atherosclerosis

Triglyceride (TG) and total cholesterol (TC) assay

The TG level was assayed in cell lyses with a TG Quantification Kit (Abcam, Cambridge, MA, USA) and TC level was detected using a Cholesterol Assay Kit (Abcam, Cambridge, MA, USA) according to the manufacturer protocol.

Measurement of intracellular ROS production, malondialdehyde (MDA) and superoxide dismutase (SOD)

Cells were collected and washed with PBS for two times, then were suspended with DCFHDA (10mM) solution. Prepared samples were incubated at $37^{\circ} \mathrm{C}$ for $20 \mathrm{~min}$. An ROS Assay Kit (Beyotime, Shanghai, China) was used and the measurement of fluorescent intensity was performed with a fluorospectrophotometer (Hitachi, Tokyo, Japan). Cell lyses was collected and centrifuged, subsequently the supernatants were used for measuring MDA and SOD levels using a Lipid Peroxidation MDA Assay Kit (Beyotime, Shanghai, China) and a SOD Activity Assay Kit (BioVision, Milpitas, CA, USA) according to the manual protocols. The absorbance at $450 \mathrm{~nm}$ was detected using a Benchmark microplate reader (Bio-Rad, Hercules, CA, USA).

Western blot analysis

Cells lysed with RIPA buffer plus fresh protease and phosphatase inhibitors were collected. Proteins extracted were quantified with the Bradford assay (Bio-Rad Laboratories, Hercules, CA). Equal amounts of

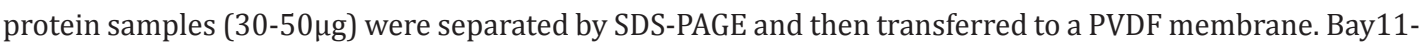

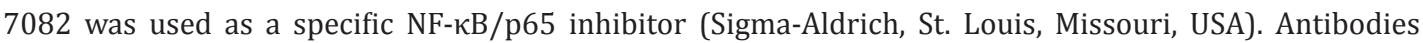

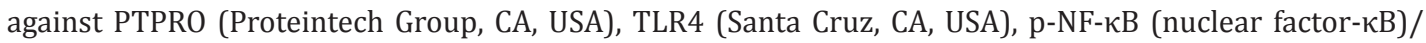
p65 (CST, Ala, USA), CD36 (CST, Ala, USA) and GAPDH (CST, Ala, USA) were used in immunoblotting. Primary antibodies were diluted 1000 times with Primary Antibody Dilution Buffer (Beyotime, Shanghai, China). Secondary antibodies (anti-rabbit) were diluted 2000 times with TBS (Tris Buffered Saline) (GeneScript, Suzhou, China). The primary antibodies were co-cultured with bands overnight at $4{ }^{\circ} \mathrm{C}$ and secondary antibodies for 1 hour at $37^{\circ} \mathrm{C}$. Immunoreactive bands were visualized using the ECL kit (Pierce, Rockford, IL, USA) and integrated density of the bands was quantified by Image software (NIH, Bethesda, MD, USA).

\section{Statistical analysis}

Data were presented as mean \pm SD. All experiments referred were performed for three times. SPSS software (SPSS Inc., Chicago, IL, USA) was used for analysis. One-way ANOVA analysis followed by LSD test was performed for multiple comparisons. $\mathrm{P}<0.05$ was considered statistically significant.

\section{Results}

OX-LDL induced cell apoptosis and PTPRO expression

RAW264.7 cells were treated with ox-LDL for $24 \mathrm{~h}$ under the concentration of $0,25,50$ and $100 \mathrm{mg} / \mathrm{L}$ or treated with ox-LDL at $50 \mathrm{mg} / \mathrm{L}$ for $0,12,24$ and $48 \mathrm{~h}$. As shown in Fig. 1, we found that cell apoptosis were promoted in a dose-dependent and time-dependent manner following ox-LDL challenging (Fig. 1A and 1B). Additionally, a dose-dependent and timedependent increase of PTPRO expression was observed (Fig. 1C and 1D). These indicated that PTPRO may be involved in the effective process induced by ox-LDL in macrophages.

PTPRO as a promoter in the formation of ox-LDL-induced macrophage-derived foam cells

To investigate the effect of PTPRO in the formation of ox-LDL-induced macrophagederived foam cells, PTPRO over-expressing RAW264.7 cells (RAW264.7-PTPRO) (Fig. 2A and 2B), negative control RAW264.7 cells (RAW264.7-NC), macrophages from PTPRO-/- mice (MC-KO) (Fig. 2A and 2B) and WT mice (MC-WT) were separately challenged with $50 \mathrm{mg} / \mathrm{L}$ ox-LDL for 24h. The results showed that ox-LDL-induced CD36 was increased when PTPRO was over-expressing and decreased after PTPRO depletion (Fig. 2C and 2D). The level of oil-red staining (Fig. 3A), TC and TG were significantly higher in PTPRO-over-expressing cells and lower in PTPRO-knockout cells (Fig. 3B and 3C). Additionally, assays delineating atherosclerosis events, macrophage-smooth muscle cells crosstalk was also performed using 
Fig. 1. Effect of ox-LDL on cell apoptosis and PTPRO expression in RAW264.7 cells. (A, C) RAW264.7 cells were stimulated with ox-LDL for $24 \mathrm{~h}$ at $0,25,50$, and $100 \mathrm{mg} / \mathrm{L}$ (B, D) RAW264.7 cells were challenged with $50 \mathrm{mg} / \mathrm{L}$ ox-LDL for 0 , 12, 24 and $48 \mathrm{~h}$. Cell apoptosis was assayed using flow cytometry. The expression levels of PTPRO and GAPDH were detected with western blotting. $(n=4)$ Data were based on at least three independent experiments. ${ }^{*} P<0.05$ vs. control group ( $0 \mathrm{~h}$ or $0 \mathrm{mg} / \mathrm{L}$ ).

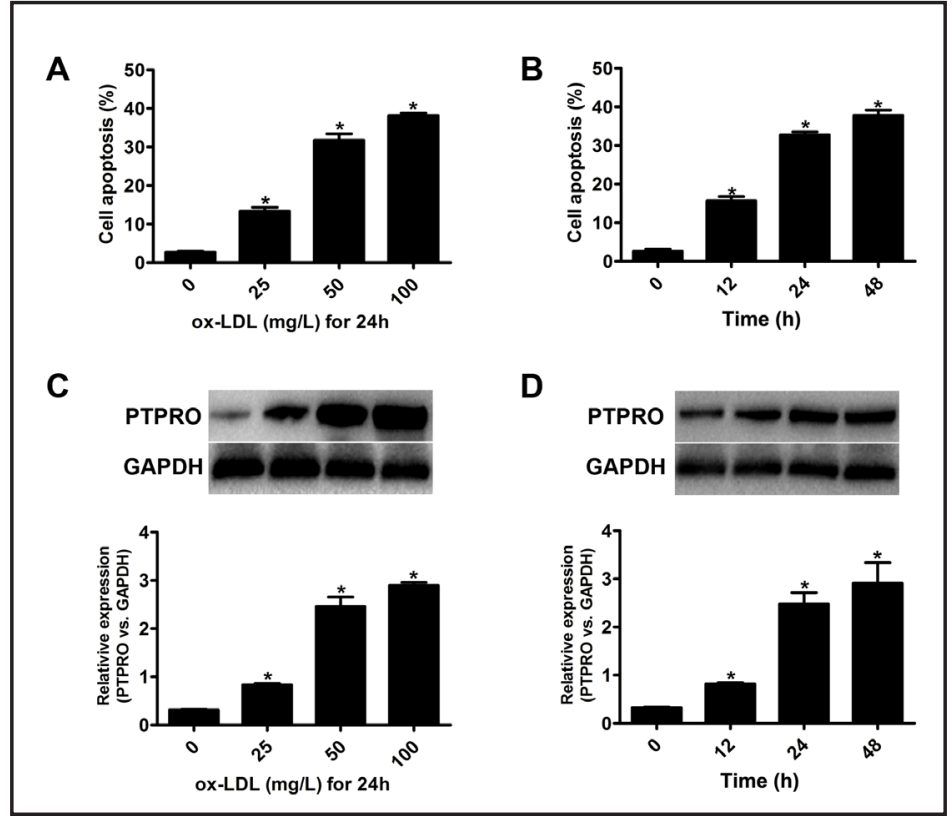

Fig. 2. The expression level of CD36 and PTPRO. (A, B) mRNA and protein levels of PTPRO were detected in PTPRO overexpressed RAW264.7 cells and in PTPRO knockout macrophages. (C, D) The expression levels of CD36 were assayed in PTPRO overexpressed cells and depleted cell after $50 \mathrm{mg} / \mathrm{L}$ ox-LDL treatment for 24h. (Macrophage, MC; WT, wild type; PTPRO, PTPRO-overexpression; NC, Negative Control plamid transfection) $(n=4)$ Data were based on at least three independent experiments. ${ }^{*} P$ $<0.05$ vs. control group. $\# P<0.05$ vs. ox-LDL group.

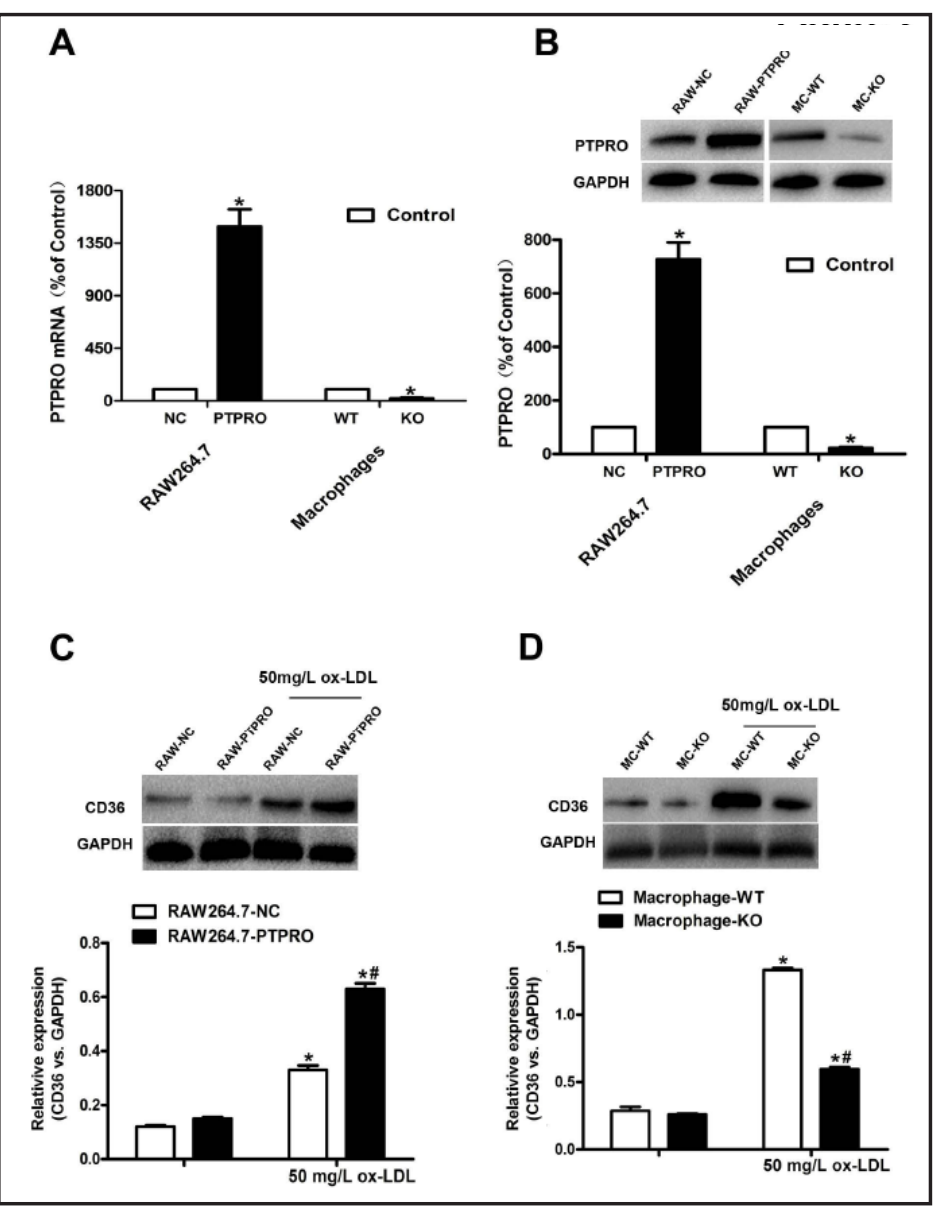

co-culture systems, which indicated that under challenge with $50 \mathrm{mg} / \mathrm{L}$ ox-LDL for 24 hours, PTPRO over-expressing RAW264,7 cells significantly inhibited the proliferation of smooth muscle cells, MOVAS. (Fig. 5) These demonstrated that PTPRO promoted the formation of 
A

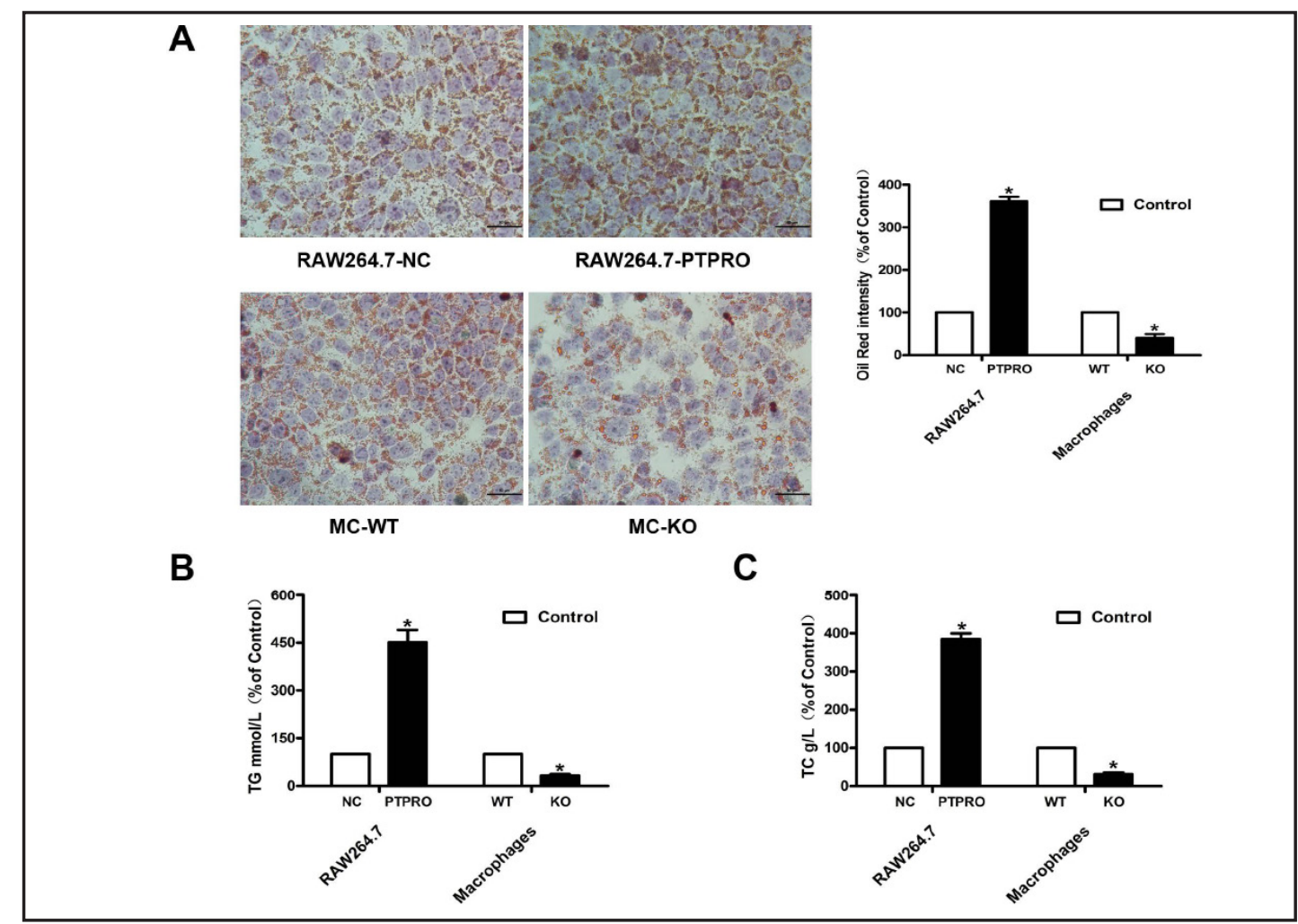

Fig. 3. Effect of PTPRO on ox-LDL-induced foam cell formation in macrophages. (A) Oil red staining in PT-

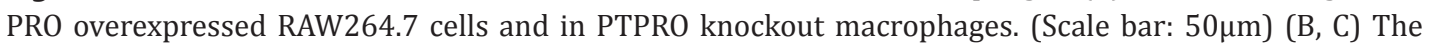
levels of TG and TC were measured as indicated. (Macrophage, MC; WT, wild type; PTPRO, PTPRO-overexpression; NC, Negative Control plamid transfection) $(n=4)$ Data were based on at least three independent experiments. ${ }^{*} P<0.05$ vs. control group.

ox-LDL-induced macrophage-derived foam cells and acted as a promoter in atherosclerosis events.

PTPRO improved ox-LDL-induced oxidative stress and macrophage apoptosis

To determine the functional roles of PTPRO in ox-LDL induced cell apoptosis and oxidative stress of macrophages, RAW264.7-PTPRO cells, RAW264.7-NC cells, MC-KO cells and MC-WT cells were exposed to $50 \mathrm{mg} / \mathrm{L}$ ox-LDL for $24 \mathrm{~h}$. As the results presented, compared to control cells, significantly elevated ROS production and MDA, but reduced SOD were observed in PTPRO-over-expressing cells (Fig. 4A, 4B and 4C). However, in PTPRO-knockout cells, higher level of SOD, but lower level of ROS production and MDA were found (Fig. 4A, 4B and 4C). The flow cytometry analysis demonstrated that cell apoptosis was improved when PTPRO was over-expressed, but decreased after PTPRO depletion (Fig. 4D), which in accordance with the expression of the classical apoptosis protein, caspase-3 (Fig. 6A). These results verified that PTPRO improved ox-LDL-induced oxidative stress and macrophage apoptosis.

PTPRO acted as a regulator in ox-LDL challenging macrophages through TLR4/NF-kB signaling pathway

As it showed in Fig. 6, under the stimulation of ox-LDL, comparing to control cells, TLR4 and NF- $\kappa B /$ p 65 phosphorylation was significantly enhanced in PTPRO over-expressing cells, and in PTPRO knockout cells, TLR4 and NF- $\kappa \mathrm{B} / \mathrm{p} 65$ phosphorylation was more significantly down-regulated (Fig. 6B and 6C). Furthermore, when specific NF- $\kappa B / p 65$ inhibitor, Bay117082 was used, up-regulation of NF- $\kappa$ B signaling was remarkably inhibited (Fig. 6B and 6C). 


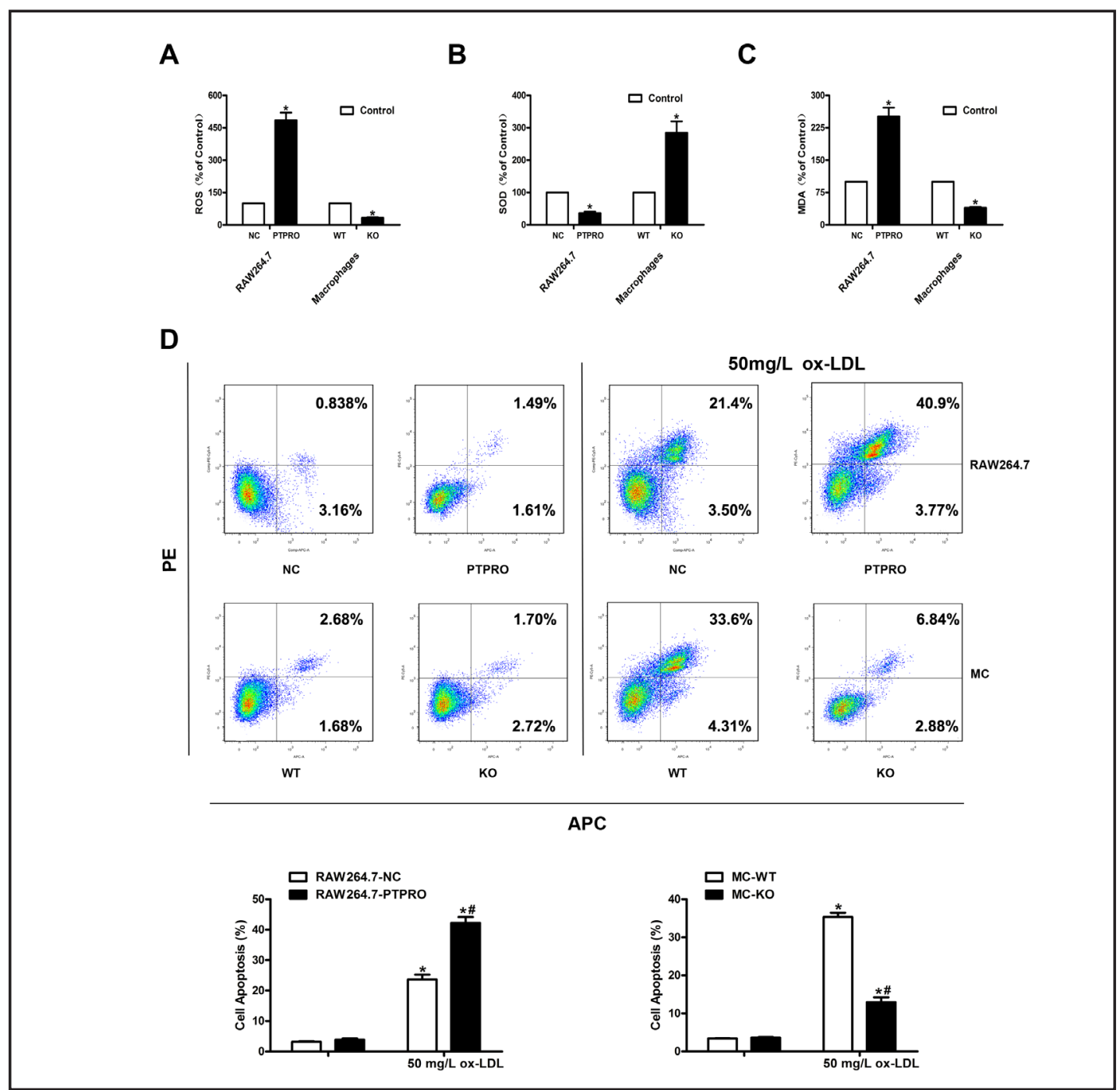

Fig. 4. Effect of PTPRO on ox-LDL-induced oxidative stress and cell apoptosis. Cells were challenged with 50 mg/L ox-LDL for $24 \mathrm{~h}$ (A, B, C) The oxidative status markers, ROS, MDA and SOD, were detected as indicated. (D) The degree of cell apoptosis was evaluated by flow cytometry. PE for nucleus staining and APC for cytomembrane staining. (Macrophage, MC; WT, wild type; PTPRO, PTPRO-overexpression; NC, Negative Control plamid transfection) $(\mathrm{n}=4)$ Data were based on at least three independent experiments. ${ }^{*} P<0.05$ vs. control group; \#P< 0.05 vs. ox-LDL group.

\section{Discussion}

In this study, we focus on the functional role of PTPRO in the development of AS. During the development of AS, persistently existing chronic inflammation finally resulted in the formation of damage within vascular endothelium, along with lipid deposition and the infiltration of inflammatory cells. Macrophages is vitally involved by swallowing over loaded lipid which in turn leaded to the disorders of metabolism and decreased anti-apoptosis ability of cells [26].

So here in this study, in order to build the AS cell model, we used moderate density of ox-LDL (50 mg/L) to challenge RAW264.7 for $24 \mathrm{~h}$. As we observed, under the treatment of ox-LDL, the expression of PTPRO was promoted in a dose-dependent and time-dependent manner, which in accordance with the cell apoptosis presenting in the similar way. This indicated that PTPRO may be a participator in regulating cell apoptosis and the development 


\section{Cellular Physiology Cell Physiol Biochem 2017;42:495-505

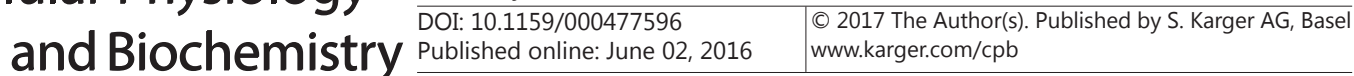

Fig. 5. The proliferation of MOVAS cells when co-cultured with RAW264.7. (A, B) The cell proliferative rates were evaluated with EdU assay. Cell nucleus was stained with DAPI. (PTPRO-overexpression; NC, Negative Control plamid transfection) $(n=4)$ Data were based on at least three independent experiments. ${ }^{*} P<0.05$ vs. not co-cultured group; \#P $<0.05$ vs. co-cultured with RAW264.7-NC group.

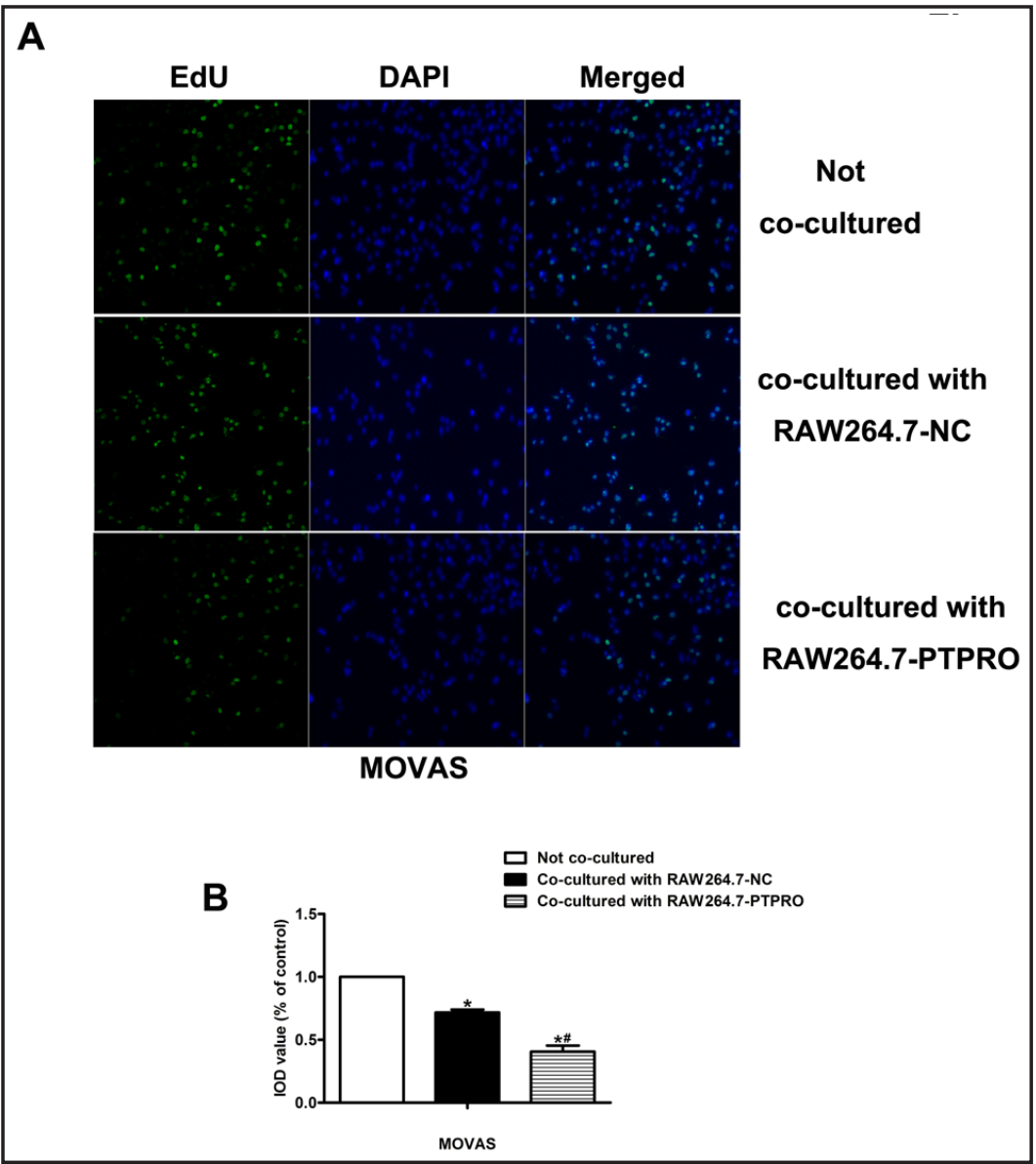

of AS. In the pathological process of AS progression, the formation of foam cells is a hallmark event. So we further investigated the effect of PTPRO in the formation of ox-LDL-induced macrophage-derived foam cells. As we found, in PTPRO over-expressing cells, the level of oil-red staining, TC and TG were significantly increased; nonetheless, adverse results were showed in PTPRO knock-out cells. And under the exposure of ox-LDL, the expression of the macrophage scavenger receptor, CD36 was accordingly regulated, higher in PTPRO over-expressing cells and lower when PTPRO was depleted. These suggested that PTPRO played vital roles in the formation of macrophage-derived foam cells. Generally, in ox-LDL induced AS, increased cell apoptosis and oxidative stress of macrophages was the main mechanism [29], so we detected that whether PTPRO functionally affected the cell apoptosis and oxidative stress of macrophages. As the results showed that over-expression of PTPRO resulted in improved cell apoptosis and oxidative stress and depletion of PTPRO reversed these effects.

In addition, we detected the expression of TLR4 in ox-LDL challenging macrophages. As we found, the level of TLR4 was significantly increased or reduced when PTPRO was overexpressing or depleted, which indicated that in ox-LDL-induced macrophage-derived foam cells, TLR4 was a downstream point of PTPRO. TLR4 is an important regulator of NF-kB pathway, which was also of great significance in the development of AS [25]. NF- $\kappa B$ is a vital transcript factor involved in inflammation, adaptive immune and innate immune. Activated NF- $\kappa B$ exacerbates inflammatory response and intensifies tissue injury through increasing pro-inflammatory and reducing anti-inflammatory cytokines. NF- $\mathrm{BB}$ can also be regulated under ox-LDL stimulation. As formerly reported, ox-LDL might upregulate IL-6 via increasing NF-kB in an IGF2-dependent way in THP-1 macrophages [24], ox-LDL stimulation can activate the TLR4/MyD88/NF-kB inflammatory signaling pathway in vascular smooth muscle cell and finally promotes the formation of foam cell [25], and in peripheral blood mononuclear 


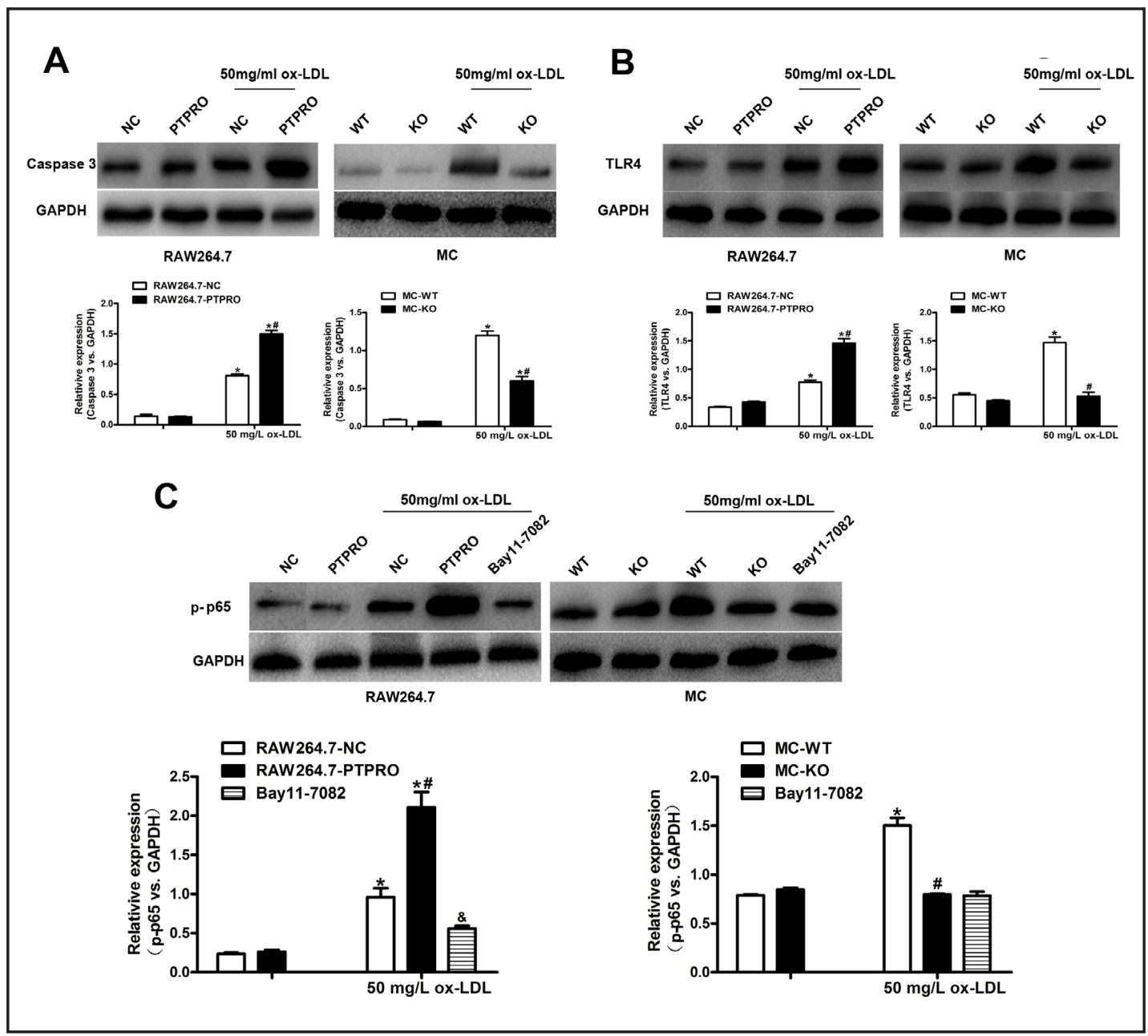

Fig. 6. Effect of PTPRO on the expression of caspase 3 and TLR4/NF-kB singnaling pathway. Investigations were performed at $24 \mathrm{~h}$. (A, B) The expression levels of caspase 3, TLR4 and GAPDH were investigated by western blot. (C) The expression levels of p-p65 and GAPDH were detected by western blot. Cells were treated with NF-kB inhibitor, Bay11-7082 (1 $\mu \mathrm{M})$ for 24 hours. (Macrophage, MC; WT, wild type; PTPRO, PTPRO-overexpression; NC, Negative Control plamid transfection) $(n=4)$ Data were based on at least three independent experiments. ${ }^{*} P<0.05$ vs. control group; $\& P<0.05$ vs. ox-LDL treated PTPRO overexpressed group; $\# P<0.05$ vs. ox-LDL group.

cells extracted from unstable angina patients, ox-LDL may induced the activation of NF$\mathrm{kB}$ [8]. PTPRO has been shown to influence the activation of transcription factors NF- $\kappa \mathrm{B}$, which mediates classical inflammatory signaling pathways in various diseases. In order to investigate the association between PTPRO/TLR4 and NF-kB, we detected the expression of NF-kB and activated NF-kB, phosphorylated IkB. As the results indicated, we demonstrated a novel PTPRO/TLR4/NF- $\kappa B$ signaling pathway in ox-LDL challenging macrophages and macrophage mediated AS. High level of activated NF- $\kappa B$ was observed in PTPRO overexpressing RAW264.7 treated with ox-LDL, which elucidated the positive relation between PTPRO and TLR4 was involved by NF- $\kappa B$ signaling pathway. However, rarely evidence was validated to clarify how PTPRO realized its functional regulation of TLR4. Further research on the exact mechanism of AS and the regulatory effects of PTPRO in AS is urgently warranted for exploration.

In conclusion, we firstly reported the functional roles of PTPRO in the AS, and further demonstrated a PTPRO/TLR4/NF-kB singnaling pathway in macrophages associated AS. In 


\section{Cellular Physiology Cell Physiol Biochem 2017;42:495-505 \\ \begin{tabular}{ll|l} 
DOI: 10.1159/000477596 & $\begin{array}{l}\text { O 2017 The Author(s). Published by S. Karger AG, Basel } \\
\text { wwww.karger.com/cpb }\end{array}$
\end{tabular}}

Liang et al.: PTPRO and Atherosclerosis

combination with previous findings, our findings further suggested that PTPRO and TLR4 may be promising therapeutic targets for AS.

\section{Acknowledgments}

This work was supported by the National Natural Science Foundation of China (81173399), the Natural Science Foundation of Jiangsu Province (BK20161115), the Nanjing medical science and technology innovation project (ZDX16013), the Jiangning science and technology project(2016Dc08), the Science and Technology Development Foundation of Nanjing Medical University (2016NJMUZD089) and the Medical Science and Technology Development Foundation of Nanjing Department of Health(YKK16235).

\section{Disclosure Statement}

Authors involved have contributed adequately to this work and have no conflicts of interest to disclose.

\section{References}

1 Taleb S: Inflammation in atherosclerosis. Arch Cardiovasc Dis 2016;16:30112-30117.

2 Zhou Y, Zhang T, Wang X, Wei X, Chen Y, Guo L, Zhang J, Wang C: Curcumin modulates macrophage polarization through the inhibition of the toll-like receptor 4 expression and its signaling pathways. Cell Physiol Biochem 2015;36:631-641.

3 Herrington W, Lacey B, Sherliker P, Armitage J, Lewington S: Epidemiology of atherosclerosis and the potential to reduce the global burden of atherothrombotic disease. Circ Res 2016;118:535-546.

4 Huang LH, Melton EM, Li H, Sohn P, Rogers MA, Mulligan-Kehoe MJ, Fiering SN, Hickey WF, Chang CC, Chang TY: Myeloid acyl-coa:Cholesterol acyltransferase 1 deficiency reduces lesion macrophage content and suppresses atherosclerosis progression. J Biol Chem 2016;291:6232-6244.

5 Huang YQ, Cai AP, Chen JY, Huang C, Li J, Feng YQ: The relationship of plasma mir-29a and oxidized low density lipoprotein with atherosclerosis. Cell Physiol Biochem 2016;40:1521-1528.

6 Tousoulis D, Psarros C, Demosthenous M, Patel R, Antoniades C, Stefanadis C: Innate and adaptive inflammation as a therapeutic target in vascular disease: The emerging role of statins. J Am Coll Cardiol 2014;63:2491-2502.

7 Moore KJ, Tabas I: Macrophages in the pathogenesis of atherosclerosis. Cell 2011;145:341-355.

8 Chistiakov DA, Orekhov AN, Bobryshev YV: Lox-1-mediated effects on vascular cells in atherosclerosis. Cell Physiol Biochem 2016;38:1851-1859.

-9 Weber C, Noels H: Atherosclerosis: Current pathogenesis and therapeutic options. Nat Med 2011;17:14101422.

10 Tedgui A, Mallat Z: Cytokines in atherosclerosis: Pathogenic and regulatory pathways. Physiol Rev 2006;86:515-581.

11 Luo C, Lian X, Hong L, Zou J, Li Z, Zhu Y, Huang T, Zhang Y, Hu Y, Yuan H, Wen T, Zhuang W, Cai B, Zhang X, Hisatome I, Yamamoto T, Huang J, Cheng J: High uric acid activates the ros-ampk pathway, impairs cd68 expression and inhibits oxldl-induced foam-cell formation in a human monocytic cell line, thp-1. Cell Physiol Biochem 2016;40:538-548.

12 Yang Y, Yang L, Liang X, Zhu G: Microrna-155 promotes atherosclerosis inflammation via targeting socs1. Cell Physiol Biochem 2015;36:1371-1381.

13 Zhang BC, Zhang CW, Wang C, Pan DF, Xu TD, Li DY: Luteolin attenuates foam cell formation and apoptosis in ox-ldl-stimulated macrophages by enhancing autophagy. Cell Physiol Biochem 2016;39:2065-2076. 


\section{Cellular Physiology Cell Physiol Biochem 2017;42:495-505 \begin{tabular}{ll|l} 
DOI: 10.1159/000477596 & $\begin{array}{l}\text { O 2017 The Author(s). Published by S. Karger AG, Basel } \\
\text { www.karger.com/cpb }\end{array}$ \\
\hline
\end{tabular}}

Liang et al.: PTPRO and Atherosclerosis

14 Kunjathoor VV, Febbraio M, Podrez EA, Moore KJ, Andersson L, Koehn S, Rhee JS, Silverstein R, Hoff HF, Freeman MW: Scavenger receptors class a-i/ii and cd36 are the principal receptors responsible for the uptake of modified low density lipoprotein leading to lipid loading in macrophages. J Biol Chem 2002;277:49982-49988.

15 Hou J, Xu J, Jiang R, Wang Y, Chen C, Deng L, Huang X, Wang X, Sun B: Estrogen-sensitive ptpro expression represses hepatocellular carcinoma progression by control of stat3. Hepatology 2013;57:678-688.

16 Hou J, Xia Y, Jiang R, Chen D, Xu J, Deng L, Huang X, Wang X, Sun B: Ptpro plays a dual role in hepatic ischemia reperfusion injury through feedback activation of nf-kappab. J Hepatol 2014;60:306-312.

17 Wang X, Yan S, Xu D, Li J, Xie Y, Hou J, Jiang R, Zhang C, Sun B: Aggravated liver injury but attenuated inflammation in ptpro-deficient mice following lps/d-gain induced fulminant hepatitis. Cell Physiol Biochem 2015;37:214-224.

18 Xu D, Wang X, Yan S, Yin Y, Hou J, Wang X, Sun B: Interaction of ptpro and tlr4 signaling in hepatocellular carcinoma. Tumour Biol 2014;35:10267-10273.

19 Dong H, Ma L, Gan J, Lin W, Chen C, Yao Z, Du L, Zheng L, Ke C, Huang X, Song H, Kumar R, Yeung SC, Zhang $\mathrm{H}$ : Ptpro represses erbb2-driven breast oncogenesis by dephosphorylation and endosomal internalization of erbb2. Oncogene 2017;36, 410-422.

20 Jiang R, Chen D, Hou J, Tan Z, Wang Y, Huang X, Wang X, Sun B: Survival and inflammation promotion effect of ptpro in fulminant hepatitis is associated with nf-kappab activation. J Immunol 2014;193:5161-5170.

-21 Choi SH, Harkewicz R, Lee JH, Boullier A, Almazan F, Li AC, Witztum JL, Bae YS, Miller YI: Lipoprotein accumulation in macrophages via toll-like receptor-4-dependent fluid phase uptake. Circ Res 2009;104:1355-1363.

-22 Stoletov K, Fang L, Choi SH, Hartvigsen K, Hansen LF, Hall C, Pattison J, Juliano J, Miller ER, Almazan F, Crosier P, Witztum JL, Klemke RL, Miller YI: Vascular lipid accumulation, lipoprotein oxidation, and macrophage lipid uptake in hypercholesterolemic zebrafish. Circ Res 2009;104:952-960.

-23 Yang K, He YS, Wang XQ Lu L, Chen QJ, Liu J, Sun Z, Shen WF: Mir-146a inhibits oxidized low-density lipoprotein-induced lipid accumulation and inflammatory response via targeting toll-like receptor 4 . FEBS Lett 2011;585:854-860.

24 Hovland A, Jonasson L, Garred P, Yndestad A, Aukrust P, Lappegard KT, Espevik T, Mollnes TE: The complement system and toll-like receptors as integrated players in the pathophysiology of atherosclerosis. Atherosclerosis 2015;241:480-494.

25 Sharma S, Garg I, Ashraf MZ: Tlr signalling and association of tlr polymorphism with cardiovascular diseases. Vascul Pharmacol 2016;87:30-37

-26 Yang K, Wang X, Liu Z, Lu L, Mao J, Meng H, Wang Y, Hu Y, Zeng Y, Zhang X, Chen Q, Liu Y, Shen W: Oxidized low-density lipoprotein promotes macrophage lipid accumulation via the toll-like receptor 4-src pathway. Circ J 2015;79:2509-2516.

27 Halloran BG, Grange JJ, So BJ, Baxter BT: Macrophage products inhibit human aortic smooth muscle cell proliferation and alter 1 alpha (i) procollagen expression. Ann Vasc Surg 1997;11:80-84.

-28 Tintut Y, Patel J, Territo M, Saini T, Parhami F, Demer LL: Monocyte/macrophage regulation of vascular calcification in vitro. Circulation 2002;105:650-655.

29 Zhang Q, Chen L, Si Z, Bu H, Narasimhulu CA, Song X, Cui MY, Liu H, Lu T, He G, Parthasarathy S, Cui L, Liu Z, Cui Y: Probucol protects endothelial progenitor cells against oxidized low-density lipoprotein via suppression of reactive oxygen species formation in vivo. Cell Physiol Biochem 2016;39:89-101. 